OPEN ACCESS

Edited by:

Alexander Yaw Debrah, Kwame Nkrumah University of Science and Technology, Ghana

Reviewed by: Upendo Mwingira, RTI International, United States Dziedzom Komi de Souza, University of Ghana, Ghana

${ }^{*}$ Correspondence: Sitara Swarna Rao Ajjampur sitararao@cmcvellore.ac.in

Specialty section: This article was submitted to Neglected Tropical Diseases,

a section of the journal

Frontiers in Tropical Diseases

Received: 16 September 2021 Accepted: 25 October 2021 Published: 26 November 2021

Citation:

Aruldas K, Khera AK and Ajjampur SSR (2021) Perspective:

Opportunities and Scope for Expanded Deworming Programs for Soil-Transmitted Helminths in India.

Front. Trop. Dis. 2:778364. doi: 10.3389/fitd.2021.778364

\section{Perspective: Opportunities and Scope for Expanded Deworming Programs for Soil-Transmitted Helminths in India}

\author{
Kumudha Aruldas ${ }^{1}$, Ajay Kumar Khera ${ }^{2}$ and Sitara Swarna Rao Ajjampur ${ }^{{ }^{*}}$ \\ 1 The Wellcome Trust Research Laboratory, Division of Gastrointestinal Sciences, Christian Medical College, Vellore, India, \\ 2 EngenderHealth, New Delhi, India
}

The current deworming strategy for soil-transmitted helminthiases (STH) focuses on preschool and school-aged children and women in the reproductive age to eliminate morbidity. However, mathematical modeling and meta-analysis show that STH could be eliminated if the entire population, including adults, were treated. The Government of India (GOI) has been deworming children through various maternal and child health programs and school health programs. One of the largest initiatives is the school-based biannual deworming program for children aged 1-19 years in the 'National Deworming Day' since 2015. Women of the reproductive age have the opportunity to get dewormed under the Anemia Free India program. Adult men and women have the opportunity to receive Albendazole in the lymphatic filariasis (LF) endemic districts. The gaps in deworming are women above the age of 49 and adult men above 19 years, living in non-LF endemic areas. Understanding gaps in deworming will help plan policy changes and program strategies for STH elimination in India, which has $21 \%$ of the global STH burden, as well as in other STH endemic countries.

Keywords: STH program, India, adult deworming, mass drug administration, community-wide MDA, elimination of STH

\section{INTRODUCTION}

Soil-transmitted helminthiases (STH) caused by Ascaris lumbricoides (roundworm), Trichuris trichiura (whipworm), and Ancylostoma duodenale or Necator americanus (hookworms) are among the most common infections in humans (1). It is estimated that about 1.45 billion people are infected with STH globally, with a high burden in India - about $21 \%$ of these STH-infected individuals live in the country (2). STH is prevalent in several states of India, with some exceeding $50 \%$ prevalence; however, most reports are based on surveys of school-aged children (SAC) alone (3). For example, the STH prevalence reported in 2015 among children aged 5-10 years in Uttar Pradesh, a highly populated state of India, was 75.6\% (69.6\% roundworm, 22.6\% hookworm, and $4.6 \%$ whipworm) (4).

The World Health Organization (WHO) recommends targeted deworming of pre-school age children (PSAC) of 1-4 years and SAC of 5-15 years, non-pregnant adolescents of 10-19 years, non- 
pregnant women in the reproductive age of 15-49 years, and pregnant women after the first trimester (1). The benefit of mass deworming has been long debated. While meta-analysis has shown that school deworming programs do not result in significant improvement in hemoglobin levels, cognition, or school performance in the short term, studies have shown a reduction in stunting among PSAC and, in the longer term, benefits on hourly earnings and standard of living as adults which validates the support for mass deworming programs (5-8). Women of reproductive age benefit from improved hemoglobin levels and a lower proportion of low birth weight babies by deworming (9-12). Given that STH control has progressed between 2008 and 2018, WHO's target of eliminating STH morbidity in pre-SAC and SAC may be achieved by 2030 with sustained STH program implementation $(13,14)$.

A systematic review and meta-analysis showed that STH reinfections could occur within three months and, at the population level, reach pre-treatment prevalence rates within a year, therefore, treating only at-risk populations may not be enough $(15,16)$. Mathematical modeling studies show that although controlling and eliminating STH infections with targeted deworming is a cost-effective approach to reduce childhood exposure and morbidity due to worm burden, it could be eliminated by treating the entire population, including adults (17-22). In real-world scenarios, a study in Cameroon showed that the distribution of Albendazole to the whole population rather than only to at-risk populations contributed to STH transmission interruption (15). A recent trial in Kenya showed that community-wide mass drug administration (cMDA) leads to a significantly higher reduction in STH prevalence in children than targeted deworming of SAC $(23,24)$. Trials are underway, including in India, to study the feasibility and impact of community-wide deworming compared to school-based deworming as a strategy for interrupting transmission of STH $(25,26)$. Empirical evidence from such trials will provide further evidence to policymakers and other key stakeholders in developing strategic STH control and elimination programs.

\section{DEWORMING PROGRAMS FOR CHILDREN AND ADOLESCENTS IN INDIA}

One of the world's largest deworming public health programs is implemented in India primarily by the Ministry of Health and Family Welfare (MoHFW), Government of India (GOI). Implementation of this is in convergence with various ministries under broader umbrella programs to improve nutrition and anemia status and control STH-related morbidity (27). The GOI targeted deworming programs, starting with deworming children and in the last 15 years, have expanded to include adolescents up to 19 years and women in the reproductive age group of 15-49 years (Figure 1). We have charted out the various schemes and stakeholders involved in deworming programs in India and identified the scope for expansion of ongoing programs to plan synergistic STH elimination strategies in the future.
The MoHFW launched the School Health Scheme in 1996-97 to address the health and nutrition needs of children included deworming, and it received fresh impetus under the National Rural Health Mission, 2005 - 2012, as a part of the Reproductive and Child Health Program, which included deworming as one of the key activities along with the provision of other health services $^{1}$. The Ministry of Women and Child Development started the Sneha Shivir Program in 2013, which focuses on undernourished children under six years of age through the Integrated Child Development Services (28). This program focused mainly on 200 districts that had a high burden of undernutrition levels. Within the districts, blocks with over $20 \%$ level of undernutrition were prioritized. This communitybased approach for preventing and managing moderate and severe malnutrition included deworming children, counseling on nutrition and childcare practices, and spot feeding of energydense food cooked at the Anganwadi centers.

The Weekly Iron and Folic Acid Supplementation (WIFS) program was launched in 2012 by MoHFW in convergence with the Ministry of Women and Child Development and Ministry of Human Resource Development to reduce the prevalence of anemia in all adolescent girls and boys in the ages $10-19$ years (29). and it mandated biannual deworming with $400 \mathrm{mg}$ of Albendazole with $100 \mathrm{mg}$ elemental iron and $500 \mu \mathrm{g}$ folic acid. The strategy was to distribute the tablets to cover 110 million adolescents girls in schools and out-of-school ${ }^{2}$. During the second phase of National Rural Health Mission, 2012-2017, child health and adolescent health received further focus when the MoHFW launched the Reproductive, Maternal, Newborn Child plus Adolescent Health program in 2013, a national program, in convergence with the Women and Child Development and the Ministry of Rural Development as well as in collaboration with various development partners (30). This encompasses biannual deworming children 6-10 years of age and adolescents in the ages 10-19 years with biannual Albendazole as part of the National Iron Plus Initiative. This scheme continued in the Rashtriya Kishor Swasthya Karyakram initiated in 2014 to respond to the health and development requirements of 243 million adolescents (10-19 years) in the country in a holistic manner in convergence with the Ministry of Women and Child Development and Ministry of Youth Affairs and Sports (31). One of the objectives of this program was to reduce malnutrition and iron deficiency anemia in adolescent girls and boys by distributing biannual Albendazole and weekly iron and folic acid.

These efforts converge as the National Deworming Day (NDD) initiative, one of the largest deworming programs of the Government (27). This was initiated in 2015 with an overall objective to control STH infections by biannual deworming of pre-school and school-aged children (1-19 years) using the platform of schools and Anganwadi centers. This program has increased its reach from nearly 89 million children in the first

\footnotetext{
${ }^{1}$ School Health Program. Accessed May 12, 2021: http://www.ihatepsm.com/blog/ school-health-program.

${ }^{2}$ Government of India. Weekly Iron and Folic Acid Supplementation Programme. Accessed May 12, 2021. https://nhm.gov.in/index1.php?lang=1\&level= $3 \&$ sublinkid $=1024 \&$ lid $=388$.
} 


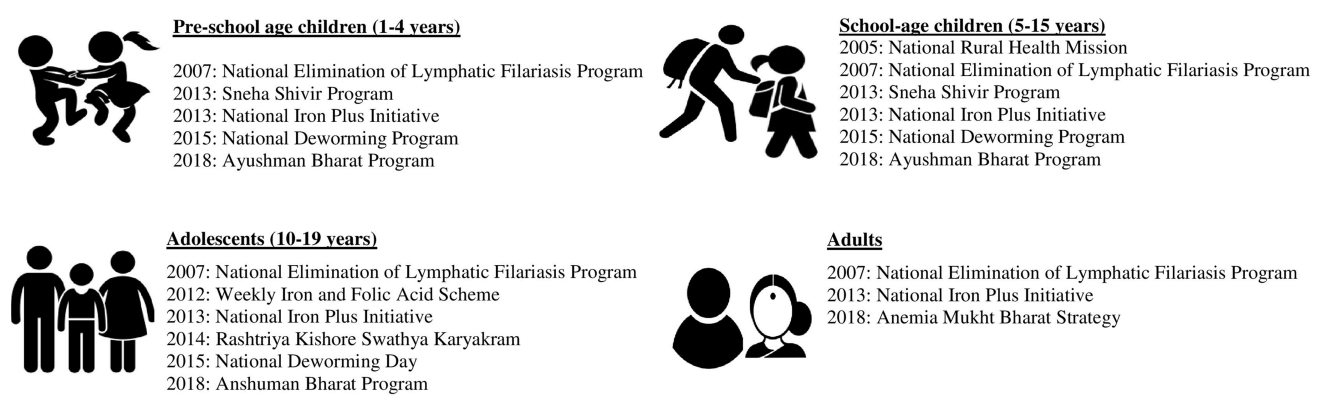

FIGURE 1 | Program initiatives in India that included deworming, 2005-2020.

round of NDD in February 2015 to almost 256 million children receiving Albendazole in the eighth round of NDD in August $2019^{3}$. In 2018, as a joint initiative of MoHFW and the Department of School Education \& Literacy, Ministry of Human Resource \& Development, the School Health Program, which included WIFS and NDD programs, was brought under the Ayushman Bharat Program (32).

\section{DEWORMING PROGRAMS FOR ADULTS IN INDIA}

Adult women were included in the deworming program along with children and adolescents in the National Iron Plus Initiative in 2013, which aimed to improve the status of anemia. This national program included deworming women in the reproductive age 15-45 years 400mg of Albendazole (33). Building on the technical and operational evidence from the Weekly Iron and Folic Acid programs and the National Iron Plus Initiative, the Anemia Mukt Bharat Strategy (Anemia Free India program) was designed in 2018, which is the intensified National Iron Plus Initiative, to reduce the prevalence of anemia among children, adolescents and women in the reproductive age group (15-49 years) by 2022 and this also includes deworming all pregnant women once in the second trimester and deworming all women in the reproductive age twice a year (34). This program is implemented at all the health system levels that women come into contact with - ranging from the Village Health and Nutrition Days at the community level to primary, secondary, and tertiary government health centers.

The scope for adult men and women of all age groups receiving Albendazole increased when the National Elimination of Lymphatic Filariasis Program started co-administration of Albendazole with Diethylcarbamazine in 2007 to interrupt transmission of filariasis (35). In this program, 400mg of Albendazole was added for all ages above two years ${ }^{4}$. As of

\footnotetext{
${ }^{3}$ Government of India. National Health Portal. Accessed November 23, 2021. https://www.nhp.gov.in/national-deworming-day_pg.

${ }^{4}$ Government of India M of H\& FW National Vector Borne Disease Control Programme. Accessed May 28, 2021. https://nvbdcp.gov.in/index4.php?lang= $1 \&$ level $=0$ \&linkid $=462 \&$ lid $=3740$.
}

2017, over a decade, the Lymphatic Filariasis (LF) program covered 256 endemic districts targeting a population of about 630 million spread over 21 states and union territories included Albendazole (36). Of these 256 districts, 94 have cleared the transmission assessment survey, and the program is continuing in 133 districts, with some receiving more than five rounds of treatment, including Albendazole. In 2018, Ivermectin was also added as Triple Drug Therapy in the Accelerated Plan of Elimination of Lymphatic Filariasis, implemented in 21 districts spread across five states ${ }^{5}$. LF programs have a synergistic effect of reducing STH prevalence (37).

\section{WATER, SANITATION, AND HYGIENE PROGRAMS AND STH}

A systematic review and meta-analysis of studies from 94 countries, including India, showed a decrease in STH infections associated with improved Water, Sanitation, and Hygiene (WASH) practices (18). Decreased odds of Ascaris infection associated with treated water and sanitation facilities were observed in Kenya (38). In contrast, cluster-randomized trials from Odisha and Madhya Pradesh conducted about a decade after the Total Sanitation Campaign launched by GOI in 1999 showed no effect of the increase in sanitation facilities on STH infections $(39,40)$. The Swatch Bharat Mission or the Clean India Mission of the Ministry of Drinking Water and Sanitation, GOI, launched in 2014, while aiming to improve WASH practices and quality of life, also aims to prevent STH infections and other enteric infections by making communities open defecation free through solid and liquid management activities. Studies show that even in households having a working toilet facility, open defecation by anyone from that household can range from $41 \%$ to $66 \%$ in some larger states like Bihar, Uttar Pradesh Madhya Pradesh, and

\footnotetext{
${ }^{5}$ Government of India NVBDCP. Elimination of Lymphatic Filariasis. Accessed June 12, 2021. https://nvbdcp.gov.in/index4.php?lang=1\&level=0\&linkid= 461 \&lid=3739.
} 
Rajasthan, indicating that more efforts on behavioral changes and acceptance are required in these communities (41).

\section{GAPS IN COMMUNITY-WIDE DEWORMING}

The GOI programs provide an opportunity for all children aged 1-19 years and WRA aged 15-49 years to be dewormed through various programs described above. In addition, the LF program provides an opportunity for adult men above the age of 19 years and women above the ages of 49 years to be dewormed in 133 endemic districts (36). The clear gap in deworming identified are adult men above age 19 years and adult women above 49 years living in non-LF program areas who are not included in any program providing deworming. This is an important gap to address in any program targeting elimination due to the ageintensity profile of hookworm infections which, unlike Ascaris and Trichuris show higher burden in the adults $(42,43)$.

\section{DISCUSSION}

As the World Health Organization (WHO), in its strategic plan document for STH 2011-2020, called for in-country coordination across ministries to maximize the deworming effort, this scoping exercise shows that GOI is highly committed to addressing STH infections by creating convergence mechanisms across various ministries $(20,21)$. Over a decade, GOI's has sustained its commitment to deworming with incorporation under various nutrition, anemia reduction, and deworming programs. Albendazole has been the drug of choice in all the deworming activities in GOI's programs and schemes. Efficacy studies conducted in seven countries, including India, showed that even a single dose of Albendazole is effective for STH infections (44).

The WHO NTD Road map 2030 calls for the elimination of STH morbidity in PSAC and SAC and among women in the reproductive age by 2030 (14). In the context of the WHO goal to eliminate STH, the GOI continues to call for collective action across various ministries and makes investments for deworming. Independent coverage surveys of the NDD program based on school reporting forms have shown high coverages in the range of $80-90 \%$ in several states (45). The impact of the program on STH prevalence in the PSAC and SAC targeted age group is also being monitored and shown a considerable decrease in prevalence ${ }^{6}$. However, both coverage and impact of deworming programs among women in reproductive age group in India are not reported and require more attention.

Although preventive chemotherapy interventions with Albendazole have reduced STH prevalence, the sustained reduction has not been observed, suggesting reinfection and ongoing transmission (46). Further, greater attention may be required in peri-urban and urban areas of India, which are

$\overline{{ }^{6} \text { Government of India. }}$ https://www.pib.gov.in/PressReleseDetailm.aspx?PRID= 1666053. estimated to be at increased risk of STH infection due to rapid urbanization and low WASH access (47). For the sustained impact of investments in deworming to prevent STH reinfection and break transmission, cMDA integrated with WASH programs would be needed $(18,23,24)$. A WASHNTD joint strategy is proposed to improve the quality of WASH programs. It is identified as one of the solutions to controlling Neglected Tropical Diseases (NTD), including STH infections (17-20).

Through all its current programs that include Albendazole, children, adolescents, WRA, and all men above the ages of 19 years in the LF districts have the opportunity to get dewormed. The GOI has allocated a budget for these programs to be delivered through schools for children and through frontline health workers in the community. The gap in the program where adults do not have the opportunity to get dewormed are adult men above 19 years and women above 49 years living in non-LF program areas. This age group also has a higher burden of hookworm infections. This is the gap India will need to address if implementing a community-wide MDA to control and eliminate STH. India has the highest STH burden globally, and therefore, it would be of value for GOI to (a) consolidate Albendazole distribution efforts across various health programs and resources allocated, (b) assess the reach of Albendazole distribution to WRA as well as estimate the population not covered by deworming across various health programs, (c) conduct an economic analysis to estimate the cost of additional drug requirement in the timeframe to elimination and cost savings if deworming could be discontinued, (d) identify any missed opportunities for all in the community to get dewormed through the various programs, (e) examine the gains of cMDA for STH elimination compared to the NDD program which is the standard of care, (f) test effectiveness of cMDA on STH prevalence, and (g) consider policy and program revision for STH elimination.

Delivery of community-wide MDA for STH in India is feasible using a two-pronged strategy, the current school-based deworming through NDD programs and community-based deworming through frontline health workers. Communitywide MDA roll-out and evaluation in states with better WASH indicators and ongoing LF programs would have a high probability of demonstrating elimination of STH and could be considered for initial phases. In conclusion, these efforts would be worthwhile due to the high burden of STH in the country as well as the potential to eliminate STH thereby being able to discontinue long running deworming programs.

\section{AUTHOR CONTRIBUTIONS}

All authors listed have made a substantial, direct, and intellectual contribution to the work, and approved it for publication.

\section{ACKNOWLEDGMENTS}

SSRA is supported by a K43/NIH Emerging Global Leader Award. 


\section{REFERENCES}

1. World Health Organization. Guideline: Preventive Chemotherapy to Control Soil-Transmitted Helminth Infections in at-Risk Population Groups. Geneva: World Health Organization (2017).

2. Pullan RL, Smith JL, Jasrasaria R, Brooker SJ. Global Numbers of Infection and Disease Burden of Soil Transmitted Helminth Infections in 2010. Parasit Vectors (2014) 7(1):1-9. doi: 10.1186/1756-3305-7-37

3. Salam N, Azam S. Prevalence and Distribution of Soil-Transmitted Helminth Infections in India. BMC Public Health (2017) 17(1):1-2. doi: 10.1186/ s12889-017-4113-2

4. Ganguly S, Barkataki S, Karmakar S, Sanga P, Boopathi K, Kanagasabai K, et al. High Prevalence of Soil-Transmitted Helminth Infections Among Primary School Children, Uttar Pradesh, India, 2015. Infect Dis Poverty (2017) 6(1):1-9. doi: 10.1186/s40249-017-0354-7

5. Taylor-Robinson DC, Maayan N, Donegan S, Chaplin M, Garner P. Public Health Deworming Programmes for Soil-Transmitted Helminths in Children Living in Endemic Areas. Cochrane Infectious Diseases Group, Editor. Cochrane Database Syst Rev (2019) 9). doi: 10.1002/14651858.CD000371.pub7

6. Lo NC, Snyder J, Addiss DG, Heft-Neal S, Andrews JR, Bendavid E. Deworming in Pre-School Age Children: A Global Empirical Analysis of Health Outcomes. PLoS Negl Trop Dis (2018) 12(5):e0006500. doi: 10.1371/ journal.pntd. 0006500

7. Hamory J, Miguel E, Walker M, Kremer M, Baird S. Twenty-Year Economic Impacts of Deworming. Proc Natl Acad Sci (2021) 118(14). doi: 10.1073/pnas.2023185118

8. Croke K, Hicks JH, Hsu E, Kremer M, Miguel E. Should the WHO Withdraw Support for Mass Deworming? PLoS Negl Trop Dis (2017) 11(6):e0005481. doi: 10.1371/journal.pntd.0005481

9. Casey GJ, Phuc TQ, MacGregor L, Montresor A, Mihrshahi S, Thach TD, et al. A Free Weekly Iron-Folic Acid Supplementation and Regular Deworming Program is Associated With Improved Hemoglobin and Iron Status Indicators in Vietnamese Women. BMC Public Health (2009) 9(1):1-8. doi: 10.1186/1471-2458-9-261

10. Thayer WM, Clermont A, Walker N. Effects of Deworming on Child and Maternal Health: A Literature Review and Meta-Analysis. BMC Public Health (2017) 17(4):113-26. doi: 10.1186/s12889-017-4747-0

11. Passerini L, Casey GJ, Biggs BA, Cong DT, Phu LB, Phuc TQ, et al. Increased Birth Weight Associated With Regular Pre-Pregnancy Deworming and Weekly Iron-Folic Acid Supplementation for Vietnamese Women. PLoS Negl Trop Dis (2012) 6(4):e1608. doi: 10.1371/journal.pntd.0001608

12. Walia B, Kmush BL, Lane SD, Endy T, Montresor A, Larsen DA. Routine Deworming During Antenatal Care Decreases Risk of Neonatal Mortality and Low Birthweight: A Retrospective Cohort of Survey Data. PLoS Negl Trop Dis (2021) 15(4):e0009282. doi: 10.1371/journal.pntd.0009282

13. Montresor A, Mupfasoni D, Mikhailov A, Mwinzi P, Lucianez A, Jamsheed M, et al. The Global Progress of Soil-Transmitted Helminthiases Control in 2020 and World Health Organization Targets for 2030. PLoS Negl Trop Dis (2020) 14(8):e0008505. doi: 10.1371/journal.pntd.0008505

14. World Health Organization. 2030 Targets for Soil-Transmitted Helminthiases Control Programmes. Geneva: World Health Organization (2019).

15. Djune-Yemeli L, Nana-Djeunga HC, Lenou-Nanga CG, Donfo-Azafack C, Domche A, Fossuo-Thotchum F, et al. Serious Limitations of the Current Strategy to Control Soil-Transmitted Helminths and Added Value of Ivermectin/Albendazole Mass Administration: A Population-Based Observational Study in Cameroon. PLoS Negl Trop Dis (2020) 14(11): e0008794. doi: 10.1371/journal.pntd.0008794

16. Jia TW, Melville S, Utzinger J, King CH, Zhou XN. Soil-Transmitted Helminth Reinfection After Drug Treatment: A Systematic Review and Meta-Analysis. PLoS Negl Trop Dis (2012) 6(5):e1621. doi: 10.1371/journal.pntd.0001621

17. Anderson R, Truscott J, Hollingsworth TD. The Coverage and Frequency of Mass Drug Administration Required to Eliminate Persistent Transmission of Soil-Transmitted Helminths. Philos Trans R Soc B: Biol Sci (2014) 369 (1645):20130435. doi: 10.1098/rstb.2013.0435

18. Strunz EC, Addiss DG, Stocks ME, Ogden S, Utzinger J, Freeman MC. Water, Sanitation, Hygiene, and Soil-Transmitted Helminth Infection: A Systematic Review and Meta-Analysis. PLoS Med (2014) 11(3):e1001620. doi: 10.1371/ journal.pmed. 1001620
19. World Health Organization. Water Sanitation \& Hygiene for Accelerating and Sustaining Progress on Neglected Tropical Diseases: A Global Strategy 20152020. Geneva: World Health Organization (2015).

20. World Health Organization. Soil-Transmitted Helminthiases: Eliminating as Public Health Problem Soil-Transmitted Helminthiases in Children: Progress Report 2001-2010 and Strategic Plan 2011-2020. Geneva: World Health Organization (2012).

21. Brooker SJ, Nikolay B, Balabanova D, Pullan RL. Global Feasibility Assessment of Interrupting the Transmission of Soil-Transmitted Helminths: A Statistical Modelling Study. Lancet Infect Dis (2015) 15 (8):941-50. doi: 10.1016/S1473-3099(15)70042-3

22. Truscott JE, Hollingsworth TD, Brooker SJ, Anderson RM. Can Chemotherapy Alone Eliminate the Transmission of Soil Transmitted Helminths? Parasit Vectors (2014) 7(1):1-8. doi: 10.1186/1756-3305-7-266

23. Clarke NE, Clements AC, Doi SA, Wang D, Campbell SJ, Gray D, et al. Differential Effect of Mass Deworming and Targeted Deworming for SoilTransmitted Helminth Control in Children: A Systematic Review and MetaAnalysis. Lancet (2017) 389(10066):287-97. doi: 10.1016/S0140-6736(16) 32123-7

24. Pullan RL, Halliday KE, Oswald WE, Mcharo C, Beaumont E, Kepha S, et al. Effects, Equity, and Cost of School-Based and Community-Wide Treatment Strategies for Soil-Transmitted Helminths in Kenya: A Cluster-Randomised Controlled Trial. Lancet (2019) 393(10185):2039-50. doi: 10.1016/S0140-6736 (18)32591-1

25. Ásbjörnsdóttir KH, Ajjampur SSR, Anderson RM, Bailey R, Gardiner I, Halliday KE, et al. Assessing the Feasibility of Interrupting the Transmission of Soil-Transmitted Helminths Through Mass Drug Administration: The DeWorm3 Cluster Randomized Trial Protocol. PLoS Negl Trop Dis (2018) 12(1):e0006253. doi: 10.1371/journal.pntd.0006166

26. Clarke NE, Ng-Nguyen D, Traub RJ, Clements ACA, Halton K, Anderson RM, et al. A Cluster-Randomised Controlled Trial Comparing School and Community-Based Deworming for Soil Transmitted Helminth Control in School-Age Children: The CoDe-STH Trial Protocol. BMC Infect Dis (2019) 19(1):1-0. doi: 10.1186/s12879-019-4449-6

27. Government of India. National Deworming Day: Operational Guidelines (2015). Available at: https://www.nhp.gov.in/national-deworming-day_pg (Accessed January 22, 2021).

28. Government of India. Operational Guidelines for Sneha Shivir-Community Based Care Through ICDS (2013). Available at: https://icdswcd.nic.in/icdsimg/ Strengthening070114.pdf (Accessed February 18, 2021).

29. Government of India. Operational Framework: Weekly Iron and Folic Acid Supplementation Programme for Adolescents RCH-Dc (2012). Available at: http://nhm.gov.in/images/pdf/programmes/wifs/operational-frameworkwifs/operational_framework_wifs.pdf (Accessed January 22, 2021).

30. Government of India. A Strategic Approach to Reproductive, Maternal, Newborn, Child and Adolescent Health (RMNCH+A) in India (2013). Available at: https://nhm.gov.in/images/pdf/RMNCH+A/RMNCH+A_ Strategy.pdf (Accessed February 18, 2021).

31. Government of India. Operational Framework for Rashtriya Kishor Swasthya Karyakram: Translating Strategy Into Programmes (2014). Available at: http:// nhm.gov.in/images/pdf/programmes/RKSK/RKSK_Operational_Framework. pdf (Accessed February 18, 2021).

32. Government of India. Operational Guidelines on School Health Programme Under Ayushman Bharat (2018). Available at: https://nhm.gov.in/New Updates_2018/NHM_Components/RMNCHA/AH/guidelines/Operational_ guidelines_on_School_Health_Programme_under_Ayushman_Bharat.pdf (Accessed January 22, 2021).

33. Government of India. Guidelines for Control of Iron Deficiency Anaemia: Nation Iron+ Initiative (2013). Available at: https://www.nhm.gov.in/images/ pdf/programmes/child-health/guidelines/Control-of-Iron-DeficiencyAnaemia.pdf (Accessed February 18, 2021).

34. Government of India. INTENSIFIED NATIONAL IRON PLUS INITIATIVE (I-NIPI): Operational Guidelines (2018). Available at: https:// anemiamuktbharat.info/wp-content/uploads/2019/09/Anemia-Mukt-BharatBrochure_English.pdf (Accessed February 18, 2021).

35. Government of India. National Roadmap for Elimination of Lymphatic Filariasis (2018). Available at: https://nvbdcp.gov.in/WriteReadData/l892s/ National-Roadmap-ELF.pdf (Accessed February 18, 2021). 
36. Government of India M of H\& FW. Accelerated Plan for Eliminate of Lymphatic Filariasis. Available at: https://nvbdcp.gov.in/WriteReadData/ 1892s/1031567531528881007.pdf (Accessed May 28, 2021).

37. Mani TR, Rajendran R, Sunish IP, Munirathinam A, Arunachalam N, Satyanarayana K, et al. Effectiveness of Two Annual, Single-Dose Mass Drug Administrations of Diethylcarbamazine Alone or in Combination With Albendazole on Soil-Transmitted Helminthiasis in Filariasis Elimination Programme. Trop Med Int Health (2004) 9(9):1030-5. doi: $10.1111 / j .1365-3156.2004 .01298 . x$

38. Pikering AJ, Njenga SM, Steinbaum L, Swarthout J, Lin A, Arnold BF, et al. Effects of Single and Integrated Water, Sanitation, Handwashing, and Nutrition Interventions on Child Soil-Transmitted Helminth and Giardia Infections: A Cluster-Randomized Controlled Trial in Rural Kenya. PLoS Med (2019) 16(6):e1002841. doi: 10.1371/journal.pmed.1002841

39. Clasen T, Boisson S, Routray P, Torondel B, Bell M, Cumming O, et al. Effectiveness of a Rural Sanitation Programme on Diarrhoea, SoilTransmitted Helminth Infection, and Child Malnutrition in Odisha, India: A Cluster-Randomised Trial. Lancet Global Health (2014) 2(11):e645-53. doi: 10.1016/S2214-109X(14)70307-9

40. Patil SR, Arnold BF, Salvatore AL, Briceno B, Ganguly S, Colford JM, et al. The Effect of India's Total Sanitation Campaign on Defecation Behaviors and Child Health in Rural Madhya Pradesh: A Cluster Randomized Controlled Trial. PLoS Med (2014) 11(8):e1001709. doi: 10.1371/journal.pmed.1001709

41. Coffey D, Gupta A, Hathi P, Khurana N, Srivastav N, Vyas S, et al. Open Defecation: Evidence From a New Survey in Rural North India. Econ Polit Wkly (2014) XLIX(38):43-55.

42. Ajjampur SS, Kaliappan SP, Halliday KE, Palanisamy G, Farzana J, Manuel M, et al. Epidemiology of Soil Transmitted Helminths and Risk Analysis of Hookworm Infections in the Community: Results From the DeWorm3 Trial in Southern India. PLoS Negl Trop Dis (2021) 15(4):e0009338. doi: 10.1371/ journal.pntd.0009338

43. Anderson RM, Turner HC, Truscott JE, Hollingsworth TD, Brooker SJ. Should the Goal for the Treatment of Soil Transmitted Helminth (STH) Infections be Changed From Morbidity Control in Children to Community-
Wide Transmission Elimination? PLoS Negl Trop Dis (2015) 9(8):e0003897. doi: 10.1371/journal.pntd.0003897

44. Vercruysse J, Behnke JM, Albonico M, Ame SM, Angebault C, Bethony JM, et al. Assessment of the Anthelmintic Efficacy of Albendazole in School Children in Seven Countries Where Soil-Transmitted Helminths Are Endemic. PLoS Negl Trop Dis (2011) 5(3):e948. doi: 10.1371/journal.pntd. 0000948

45. Evidence Action. Independent Monitoring of National Deworming Day in Chhattisgarh: Report (2018). Available at: https://files.givewell.org/files/ DWDA\%202009/DtWI/Deworm_the_World_Chhattisgarh_NDD_PMCV_ report_August_2018.pdf (Accessed September 16, 2021).

46. Abraham D, Kaliappan S, Walson J, Rao Ajjampur S. Intervention Strategies to Reduce the Burden of Soil-Transmitted Helminths in India. Indian J Med Res (2018) 147(6):533.

47. Pullan RL, Brooker SJ. The Global Limits and Population at Risk of SoilTransmitted Helminth Infections in 2010. Parasit Vectors (2012) 5(1):1-4. doi: $10.1186 / 1756-3305-5-81$

Conflict of Interest: The authors declare that the research was conducted in the absence of any commercial or financial relationships that could be construed as a potential conflict of interest.

Publisher's Note: All claims expressed in this article are solely those of the authors and do not necessarily represent those of their affiliated organizations, or those of the publisher, the editors and the reviewers. Any product that may be evaluated in this article, or claim that may be made by its manufacturer, is not guaranteed or endorsed by the publisher.

Copyright (๑) 2021 Aruldas, Khera and Ajjampur. This is an open-access article distributed under the terms of the Creative Commons Attribution License (CC BY). The use, distribution or reproduction in other forums is permitted, provided the original author(s) and the copyright owner(s) are credited and that the original publication in this journal is cited, in accordance with accepted academic practice. No use, distribution or reproduction is permitted which does not comply with these terms. 